\title{
Immobile Indices and CQ-Free Optimality Criteria for Linear Copositive Programming Problems
}

\section{O. I. Kostyukova, T. V. Tchemisova \& O. S. Dudina}

Set-Valued and Variational Analysis

Theory and Applications

ISSN 1877-0533

Volume 28

Number 1

Set-Valued Var. Anal (2020) 28:89-107

DOI 10.1007/s11228-019-00527-y 
Your article is protected by copyright and all rights are held exclusively by Springer Nature B.V.. This e-offprint is for personal use only and shall not be self-archived in electronic repositories. If you wish to self-archive your article, please use the accepted manuscript version for posting on your own website. You may further deposit the accepted manuscript version in any repository, provided it is only made publicly available $\mathbf{1 2}$ months after official publication or later and provided acknowledgement is given to the original source of publication and a link is inserted to the published article on Springer's website. The link must be accompanied by the following text: "The final publication is available at link.springer.com". 


\title{
Immobile Indices and CQ-Free Optimality Criteria for Linear Copositive Programming Problems
}

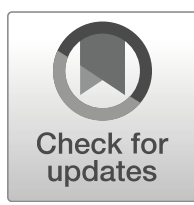

\author{
O. I. Kostyukova ${ }^{1} \cdot$ T. V. Tchemisova ${ }^{2}$ - O. S. Dudina $a^{3,4}$
}

Received: 28 November 2018 / Accepted: 20 December 2019 / Published online: 27 January 2020

(C) Springer Nature B.V. 2020

\begin{abstract}
We consider problems of linear copositive programming where feasible sets consist of vectors for which the quadratic forms induced by the corresponding linear matrix combinations are nonnegative over the nonnegative orthant. Given a linear copositive problem, we define immobile indices of its constraints and a normalized immobile index set. We prove that the normalized immobile index set is either empty or can be represented as a union of a finite number of convex closed bounded polyhedra. We show that the study of the structure of this set and the connected properties of the feasible set permits to obtain new optimality criteria for copositive problems. These criteria do not require the fulfillment of any additional conditions (constraint qualifications or other). An illustrative example shows that the optimality conditions formulated in the paper permit to detect the optimality of feasible solutions for which the known sufficient optimality conditions are not able to do this. We apply the approach based on the notion of immobile indices to obtain new formulations of regularized primal and dual problems which are explicit and guarantee strong duality.
\end{abstract}

Keywords Semi-infinite programming · Copositive programming ·

Optimality conditions · Constraint qualification - Normalized immobile index set ·

Strong duality

Mathematics Subject Classification (2010) 90C25 · 90C34 · 90C46 · 49N15

T. V. Tchemisova

tatiana@ua.pt

O. I. Kostyukova

kostyukova@im.bas-net.by

O. S. Dudina

dudina@bsu.by

1 Institute of Mathematics, National Academy of Sciences of Belarus, Surganov str. 11, 220072, Minsk, Belarus

2 CIDMA-Center for Research and Development in Mathematics and Applications, Department of Mathematics, University of Aveiro, Campus Universitário Santiago, 3810-193, Aveiro, Portugal

3 Belarusian State University, Independence Ave., 4, Minsk, Belarus

4 RUDN University, 6, Miklukho-Maklaya st., 117198, Moscow, Russia 


\section{Introduction}

Given an integer $p>1$, denote by $\mathcal{S}^{p}$ the vector space of real symmetric $p \times p$-matrices and by $\mathbb{R}_{+}^{p}$ the set of element-wise nonnegative $p$-vectors. Consider the cone of $p \times p$ copositive matrices

$$
\mathcal{C O P}=\mathcal{C O P}^{p}:=\left\{A \in \mathcal{S}^{p}: t^{\top} A t \geq 0 \forall t \in \mathbb{R}_{+}^{p}\right\} .
$$

In the paper, we consider a linear copositive programming problem in the form

$$
\sup _{x \in \mathbb{R}^{n}} c^{\top} x \quad \text { s.t. } \sum_{m=1}^{n} A_{m} x_{m}+A_{0} \in \mathcal{C O} \mathcal{P},
$$

where $x=\left(x_{m}, m=1, \ldots, n\right)^{\top}$ is a vector of decision variables $x_{m} ; c \in \mathbb{R}^{n}$ and $A_{m} \in$ $\mathcal{S}^{p} \forall m=0,1, \ldots, n$, are given vector and matrices.

Linear copositive programing can be considered as a generalization of semidefinite programming (SDP) since the optimization there is performed over the cone $\mathcal{C O P}$ of matrices that are positive semi-definite on the non-negative orthant $\mathbb{R}_{+}^{p}$.

Copositive programming is a relatively new field of conic optimization, that has been actively developing in recent years. Copositive models arise in non-convex quadratic programming with linear and binary constraints $[8,14]$, graph theory and combinatorics [11, 32], among others. The diversity of copositive formulations in different domains of optimization (continuous and discrete, deterministic and stochastic, robust optimization with uncertainty and others) is described in [5, 6, 14, 39]. According to [12], the fact that many combinatorial problems admit copositive formulations "is remarkable since it provides a convex formulation of many NP-hard problems".

Although the copositive problems are convex, they are known to be NP-hard, with difficulties caused by the copositive cone constraint since testing of copositivity of matrices is co-NP-complete [33].

The dual copositive problems are formulated over the cone of completely positive matrices, $\mathcal{C P}$, which are also hard to identify. The matrix cones $\mathcal{C O} \mathcal{P}$ and $\mathcal{C P}$ possess highly interesting properties and during many years attract interest in the linear algebra community. For the studies of these properties, we refer the interested reader to $[4,12,13,16,18]$, and the references therein.

Optimality conditions are an important issue when exploring any optimization problem, as they do not only allow to test the optimality of a given feasible solution, but also to develop efficient methods for numerical solving the problem. Optimality conditions are usually formulated specifically for certain classes of optimization problems, which makes it possible to use more efficiently the certain features of these problems, properties of their objective and constraint functions, as well as structure of feasible sets. Often, optimality conditions use different constraint qualifications (CQs) (see e.g. [7, 29, 41], and the references therein). Testing CQs is not always an easy task, and, at the same time, the fail of CQs can lead to numerical difficulties such as increase of the expected number of iterations, the absence of an adequate stopping rule and even to incorrect solutions. Therefore the search for optimality conditions that do not use any CQ (CQ-free optimality conditions) is an important challenge in Optimization from both theoretical and practical points of view.

In linear copositive programming, the usual approach to optimality conditions is that the given copositive problem is reformulated as an equivalent problem of semi-infinite programming (SIP), and then optimality conditions for the copositive problems are deduced from such conditions for their semi-infinite equivalents (see e.g. [1, 23]). This allows one to 
use a rich arsenal of optimal conditions known in SIP. Extensive bibliography is devoted to optimality conditions for SIP and their applications (see [7, 15, 17, 28, 30, 39, 40] and the references therein).

In our previous papers [19-24] and others, we introduced notions of immobile indices and their immobility orders for different classes of convex optimization problems and formulated for these problems new CQ-free optimality conditions. These optimality conditions permit to test optimality of a given feasible solution even in the cases when other known conditions fail. Algorithms that permit to find the immobile indices in a finite number of steps were described and justified for problems of SIP and SDP.

In [23], we reformulated the notion of immobile indices for linear copositive problems and used the set of immobile indices to obtain new optimality conditions for these problems. These conditions do not use any CQs, but are formulated under a less restrictive assumption about a finite number of immobile indices.

The new approach based on the immobile indices permitted also to obtain strong dual formulations for copositive problems. The new regularized dual problem was formulated for linear copositive problem under the finiteness assumption and it was proved that the gap between the optimal values of the primal and the regularized dual copositive problems was null.

In the present paper, we continue to study the linear copositive problems and discover new properties of the feasible sets and the sets of immobile indices. In particular, we introduce a normalized immobile index set and show that this set either is empty or is a union of a finite number of closed convex polyhedra. These results permit to prove new optimality conditions without any CQs as well as without any other special assumptions about the set of immobile indices (such as its finiteness). Moreover, now we do not need anymore to find all elements of the normalized immobile index set (the immobile indices), and construct our new optimality conditions only basing on the extremal points (vertices) of the convex hull of this set. We consider an illustrative example showing that the optimality conditions obtained in the paper permit to detect optimality of feasible solutions for which other known sufficient optimality conditions are not able to do this. Further, we present new formulations of regularized primal and dual copositive problems which are explicit and guarantee the strong duality.

The optimality and duality results of the paper may be used for constructing efficient numerical methods for copositive programming. Due to high difficulty of copositive problems, this area is not explored yet. Therefore the topics raised in the article are of great importance and relevance.

It is worthwhile to mention that an important contribution to the study of regularity and stability of optimization problems was done by Alexander Kruger. In [29], together with his co-authors he studied new relaxations of the Mangasarian-Fromovitz CQ and examined their relationship with some standard CQs for nonlinear optimization problems. Optimality conditions for different classes of optimization problems were studied in [25, 26, 28]. Several papers by A.Kruger are dedicated to the study of semi-infinite programming problems and different aspects connected with semi-infinite collections of sets (see e.g. [10, 27, 30, $34,35])$.

The paper is organized as follows. In Section 2, we state a linear copositive problem and introduce an equivalent SIP formulation. Having defined immobile indices and a normalized immobile index set, we devote the rest of the section to the study of the structure and properties of this set. In Section 3, we prove new optimality criteria for linear copositive problems. An illustrative example is discussed in Section 4. A regularized pair of primal and dual problems in a conic form is formulated in Section 5. It is proved that the strong duality 
is guaranteed for this pair of dual problems. Section 6 contains some conclusions and final remarks.

\section{Linear Copositive Programming Problem}

\subsection{Problem Statement and an Equivalent Semi-Infinite Formulation}

Consider a linear copositive problem in the form

$$
\min _{x \in \mathbb{R}^{n}} c^{\top} x \quad \text { s.t. } t^{\top} \mathcal{A}(x) t \geq 0 \quad \forall t \in \mathbb{R}_{+}^{p},
$$

where $x=\left(x_{1}, \ldots, x_{n}\right)^{\top}$ is a vector of decision variables; $t=\left(t_{1}, \ldots, t_{p}\right)^{\top}$ is a $p$-vector of indices, the constraints contain a matrix function $\mathcal{A}(x)$ in the form

$$
\mathcal{A}(x):=\sum_{m=1}^{n} A_{m} x_{m}+A_{0},
$$

matrices $A_{m} \in \mathcal{S}^{p}, m=0,1, \ldots, n$ and vector $c \in \mathbb{R}^{n}$ are given.

It is well known that the linear copositive problem (2) is equivalent to the following problem of convex SIP:

$$
\min _{x} c^{\top} x \text { s.t. } t^{\top} \mathcal{A}(x) t \geq 0 \quad \forall t \in T
$$

with a $p$ - dimensional compact index set in the form of the simplex

$$
T:=\left\{t \in \mathbb{R}_{+}^{p}: \mathbf{e}^{\top} t=1\right\} .
$$

Here $\mathbf{e}=(1,1, \ldots, 1)^{\top} \in \mathbb{R}^{p}$

Denote by $X$ the set of feasible solutions of problems (2) and (3):

$$
X:=\left\{x \in \mathbb{R}^{n}: t^{\top} \mathcal{A}(x) t \geq 0 \quad \forall t \in \mathbb{R}_{+}^{p}\right\} .
$$

Notice that the set $X$ is convex.

According to the common definitions (see, e.g. [7]), the constraints of the SIP problem (3) satisfy the Slater regularity condition (the Slater CQ) if

$$
\exists \bar{x} \in \mathbb{R}^{n} \text { such that } t^{\top} \mathcal{A}(\bar{x}) t>0 \forall t \in T,
$$

and the constraints of the linear copositive problem (2) satisfy the Slater condition if

$$
\exists \bar{x} \in \mathbb{R}^{n} \text { such that } t^{\top} \mathcal{A}(\bar{x}) t>0 \forall t \in \mathbb{R}_{+}^{p} \backslash\{\mathbf{0}\} .
$$

Following [23, 24], let us define the sets of immobile indices $R_{i m}$ and $T_{i m}$ in the equivalent problems (2) and (3), respectively:

$$
\begin{aligned}
& R_{i m}:=\left\{t \in \mathbb{R}_{+}^{p}: t^{\top} \mathcal{A}(x) t=0 \quad \forall x \in X\right\} \text { and } \\
& T_{i m}:=\left\{t \in T: t^{\top} \mathcal{A}(x) t=0 \quad \forall x \in X\right\} .
\end{aligned}
$$

It is evident that the sets $R_{i m}$ and $T_{i m}$ are closely related:

$$
\begin{aligned}
& R_{i m}=\left\{t \in \mathbb{R}^{p}: t=\alpha \tau, \tau \in T_{i m}, \alpha \geq 0\right\} \text { and } \\
& T_{i m}=\left\{t \in R_{i m}: \mathbf{e}^{\top} t=1\right\} .
\end{aligned}
$$

The following proposition is a corollary of Proposition 2 from [24].

Proposition 1 1. Given an SIP problem in the form (3), the Slater condition (4) is equivalent to the emptiness of the index set $T_{i m}$. 
2. Given a linear copositive problem in the form (2), the Slater condition (5) is equivalent to the condition $R_{\text {im }}=\{\mathbf{0}\}$.

3. The interrelated problems (2) and (3) satisfy (or not satisfy) the Slater condition simultaneously.

It follows from relations (6) and Proposition 1 that we can consider the set $T_{i m}$ as a normalized immobile index set of the original problem (2). In what follows, we will mainly consider this set instead of the set $R_{i m}$.

Let $\mathcal{C P}$ denote the set of completely positive $p \times p$ matrices:

$$
\mathcal{C P}=\mathcal{C P}^{p}:=\operatorname{conv}\left\{t t^{\top}: t \in \mathbb{R}_{+}^{p}\right\} .
$$

Here and on, conv $\mathcal{D}$ denotes the convex hull of a set $\mathcal{D}$ and $A \bullet B:=\langle A, B\rangle$ stays for the trace inner product of matrices $A, B \in \mathcal{S}^{p}$.

Notice that $\mathcal{C P}$ is the dual (polar) cone to the cone $\mathcal{C O P}$ of copositive matrices (see [1, 6] et al.).

Let us say that a feasible solution $x^{0} \in X$ satisfies the Karush-Kuhn-Tucker (KKT) conditions if there exists a matrix $\Omega \in \mathcal{C P}$ such that

$$
(\mathrm{KKT}): \quad-c_{m}+\Omega \bullet A_{m}=0, m=1, \ldots, n, \Omega \bullet \mathcal{A}\left(x^{0}\right)=0 .
$$

Following [1], the optimality conditions for problem (2) can be formulated in the form of the theorem.

Theorem 1 If a feasible solution $x^{0} \in X$ satisfies the conditions (KKT), then $x^{0}$ is a minimizer of problem (2). On the other hand, under the Slater condition (5) a minimizer $x^{0}$ of problem (2) must satisfy (KKT).

If $T_{i m}=\varnothing$, then, according to Proposition 1, the constraints of problem (2) satisfy the Slater condition and in this case the conditions (KKT) are not only sufficient but also necessary optimality conditions for linear copositive programming. If $T_{i m} \neq \emptyset$, then Theorem 1 is not a criterion.

The aim of this paper is to obtain for problem (2) new optimality conditions that do not require the Slater condition or any other additional condition to be satisfied. Therefore, in what follows, we will not impose the condition $T_{i m}=\emptyset$ and, moreover, will focus on the case when this condition is not fulfilled.

\subsection{The Structure of the Normalized Immobile Index Set}

The next lemma shows that the normalized immobile index set of problem (2) has a specific structure. We will use this result in our study.

Lemma 1 Given the linear copositive problem (2), the normalized immobile index set $T_{i m}$ is either empty or can be represented as a union of a finite number of convex closed bounded polyhedra.

Proof Set $P:=\{1,2, \ldots, p\}$ and suppose that $T_{i m} \neq \emptyset$.

For $t \in T_{i m}$, introduce a set $P_{+}(t):=\left\{k \in P: t_{k}>0\right\}$.

Denote: $\mathcal{P}=\left\{\mathcal{I} \subset P: \exists t \in T_{i m}, \mathcal{I}=P_{+}(t)\right\}$. From the definition of the set $\mathcal{P}$, it follows that $|\mathcal{P}| \leq 2^{p}-1$. 
For any set $\mathcal{I} \in \mathcal{P}$, consider the corresponding set $T_{+}(\mathcal{I}):=\operatorname{cl}\left\{t \in T_{i m}: P_{+}(t)=\mathcal{I}\right\}$, where $\operatorname{cl}(\mathcal{D})$ stays for the closure of a set $\mathcal{D}$. It is evident that

$$
T_{i m}=\bigcup_{\mathcal{I} \in \mathcal{P}} T_{+}(\mathcal{I})
$$

Given $\mathcal{I} \in \mathcal{P}$, consider a set of indices

$$
\left\{t^{s} \in T_{+}(\mathcal{I}), s \in S=S(\mathcal{I})\right\} \text { with } 0<|S| \leq p,
$$

such that $|S|=\operatorname{rank}\left(t^{s}, s \in S\right)=\operatorname{rank}\left(t \in T_{+}(\mathcal{I})\right)$.

By construction, for any $t \in T_{+}(\mathcal{I})$, we have

$$
P_{+}(t) \subset \mathcal{I} ; e_{k}^{\top} \mathcal{A}(x) t=0 \forall k \in \mathcal{I}, \forall x \in X,
$$

where $e_{k}$ is the $k$-th vector of the canonical basis of $\mathbb{R}^{p}$. Hence, for all $\tau \in T_{+}(\mathcal{I})$ and all $t \in T_{+}(\mathcal{I})$, the following equalities hold:

$$
\tau^{\top} \mathcal{A}(x) t=\sum_{k \in P_{+}(\tau)} \tau_{k} e_{k}^{\top} \mathcal{A}(x) t=\sum_{k \in \mathcal{I}} \tau_{k} e_{k}^{\top} \mathcal{A}(x) t=0 \forall x \in X .
$$

Consequently,

$$
\begin{aligned}
& \left(t^{s}\right)^{\top} \mathcal{A}(x) t^{j}=0 \quad \forall s \in S, \quad \forall j \in S \Longrightarrow \\
& \tau^{\top} \mathcal{A}(x) t=0 \quad \forall \tau \in M(S) \quad \forall t \in M(S), \quad \forall x \in X,
\end{aligned}
$$

where $M(S):=\operatorname{span}\left\{t^{s}, s \in S\right\}$.

Given $\mathcal{I} \in \mathcal{P}$, consider the set $T_{i m}(\mathcal{I}):=T \cap M(S)$. By construction, $T_{i m}(\mathcal{I})$ is a convex closed bounded polyhedron and it follows from (8) that

$$
T_{i m}(\mathcal{I}) \subset T_{i m} .
$$

Suppose that $t \in T_{+}(\mathcal{I})$. Then $t \geq 0, \mathbf{e}^{\top} t=1$, and $t=\sum_{s \in S} \alpha_{s} t^{s}$. Hence, $t \in M(S)$ and, consequently, $t \in T_{i m}(\mathcal{I})$. Thus, we have shown the inclusion

$$
T_{+}(\mathcal{I}) \subset T_{i m}(\mathcal{I}) .
$$

It follows from (9) that

$$
\bigcup_{\mathcal{I} \in \mathcal{P}} T_{i m}(\mathcal{I}) \subset T_{i m}
$$

On the other hand, from (7) and (10) we conclude that

$$
T_{i m}=\bigcup_{\mathcal{I} \in \mathcal{P}} T_{+}(\mathcal{I}) \subset \bigcup_{\mathcal{I} \in \mathcal{P}} T_{i m}(\mathcal{I}) .
$$

Inclusions (11) and (12) imply that $T_{i m}=\bigcup_{\mathcal{I} \in \mathcal{P}} T_{i m}(\mathcal{I})$.

Hence, we have proved that either the set $T_{i m}$ is empty or it can be represented as a union of a finite number (less or equal to $2^{p}-1$ ) of convex closed bounded polyhedra. The lemma is proved.

\subsection{The Properties of the Immobile Index Set and the Feasible Set of Problem (2)}

Here, as above, we consider a general case of problem (2) where the normalized immobile index set $T_{i m}$ may be non-empty. Let us study some properties of the set $T_{i m}$ and its convex hull conv $T_{i m}$.

Suppose that $T_{i m} \neq \emptyset$. According to the results of the previous subsection, the set $T_{i m} \subset$ $T$ is a union of a finite number of closed convex bounded polyhedra hence the set conv $T_{i m}$ is a bounded polyhedron. 
Denote by $\{\tau(j), j \in J\}$ the set of vertices of conv $T_{i m}$ :

$$
\tau(j) \in T_{i m}, \quad \tau(j)=\left(\tau_{k}(j), k \in P\right) \forall j \in J .
$$

In the case $T_{i m}=\emptyset$, we have conv $T_{i m}=\emptyset$, which means that there are no vertices in the set conv $T_{i m}$ and hence in this case we should set $J:=\emptyset$.

Proposition 2 Given problem (2) and the corresponding set (13) of vertices of the set conv $T_{i m}$, for any $x \in X$, the following relations take place:

$$
\mathcal{A}(x) \tau(j) \geq 0, \quad j \in J .
$$

The proof of the proposition follows from the definition of immobile indices and Lemma 2.6 from [2].

Consider the set

$$
\mathcal{X}:=\left\{x \in \mathbb{R}^{n}: \mathcal{A}(x) \tau(j) \geq 0 \quad \forall j \in J\right\} .
$$

Then, by Proposition 2, $X \subset \mathcal{X}$.

Proposition 3 Consider the linear copositive problem (2) with the normalized immobile index set $T_{i m}$. Let the vertices of conv $T_{i m}$ be denoted as in (13) and the set $\mathcal{X}$ be defined as in (15). Then for all $x \in \mathcal{X}$, the following relations take place:

$$
t^{\top} \mathcal{A}(x) t \geq 0 \quad \forall t \in \operatorname{conv} R_{\text {im }} .
$$

Proof Let $t \in \operatorname{conv} R_{i m}$. Then $t=\sum_{j \in J} \alpha_{j} \tau(j), \quad \alpha_{j} \geq 0 \forall j \in J$. Hence,

$$
t^{\top} \mathcal{A}(x) t=\left(\sum_{j \in J} \alpha_{j} \tau(j)\right)^{\top} \mathcal{A}(x)\left(\sum_{j \in J} \alpha_{j} \tau(j)\right)=\sum_{s \in J} \sum_{j \in J} \alpha_{s} \alpha_{j}(\tau(s))^{\top} \mathcal{A}(x) \tau(j) .
$$

Since $x \in \mathcal{X}$, then $\mathcal{A}(x) \tau(j) \geq 0$ for all $j \in J$. Taking into account that by construction $\tau(j) \in \mathbb{R}_{+}^{p} \forall j \in J$, we get $(\tau(s))^{\top} \mathcal{A}(x) \tau(j) \geq 0 \forall s \in J, \forall j \in J$. These inequalities and the conditions $\alpha_{j} \geq 0 \forall j \in J$, together with equalities (17) imply inequalities (16). The proposition is proved.

Given $\varepsilon>0$, consider the sets

$$
\begin{aligned}
& T(\varepsilon):=\left\{t \in T: \rho\left(t, \text { conv } T_{i m}\right) \geq \varepsilon\right\}, \\
& T(\varepsilon):=\left\{t \in T: \rho\left(t, \operatorname{conv} T_{i m}\right) \leq \varepsilon\right\},
\end{aligned}
$$

and

$$
\mathcal{X}(\varepsilon):=\left\{z \in \mathcal{X}: t^{\top} \mathcal{A}(z) t \geq 0 \forall t \in T(\varepsilon)\right\},
$$

where $\rho(l, B)=\min _{\beta \in B}\|l-\beta\|$ is the distance between a vector $l$ and a set $B$ in $\mathbb{R}^{p},\|y\|=$ $\sqrt{y^{\top} y}$ for $y \in \mathbb{R}^{p}$, the set $\mathcal{X}$ is defined in (15).

Lemma 2 There exists $\varepsilon_{0}>0$ such that $\mathcal{X}\left(\varepsilon_{0}\right)=X$.

Proof It follows from Proposition 2 that $X \subset \mathcal{X}(\varepsilon)$ for all $\varepsilon>0$. Let us show that there exists $\varepsilon_{0}>0$ such that $\mathcal{X}\left(\varepsilon_{0}\right) \subset X$. Suppose the contrary. Then, for all $\varepsilon>0$ there exists $z(\varepsilon) \in \mathcal{X}(\varepsilon)$ such that

$$
(t(\varepsilon))^{\top} \mathcal{A}(z(\varepsilon)) t(\varepsilon)<0,
$$

where $t(\varepsilon):=\arg \left\{\min _{t} t^{\top} \mathcal{A}(z(\varepsilon)) t\right.$, s.t. $\left.t \in T\right\}$. 
Since, by construction, it holds $t^{\top} \mathcal{A}(z(\varepsilon)) t \geq 0 \quad \forall t \in T(\varepsilon) \cup$ conv $T_{i m}$ (see Proposition 3 and the definition of the set $\mathcal{X}(\varepsilon)$ in (19)), then one can conclude that $t(\varepsilon) \in \widehat{T(\varepsilon)} \backslash$ conv $T_{i m}$. Hence there exists $t^{*}:=\lim _{\varepsilon \rightarrow+0} t(\varepsilon), t^{*} \in \operatorname{conv} T_{i m}$.

For a sufficiently small $\varepsilon>0$, let us consider the vector $l(\varepsilon):=t(\varepsilon)-t^{*}$. It is evident that $\mathbf{e}^{\top} l(\varepsilon)=0$ and for all $k \in P$, the following conditions are satisfied:

if $t_{k}^{*}=0$ then $l_{k}(\varepsilon)=t_{k}(\varepsilon) \geq 0$; if $t_{k}(\varepsilon)=0$ then $t_{k}^{*}=0$ and $l_{k}(\varepsilon)=0$.

Consequently, the direction $l(\varepsilon)$ is feasible in the set $T$ for both vectors $t^{*}$ and $t(\varepsilon)$. Hence, there exists $\gamma_{0}>1$ such that

$$
t^{*}+\gamma l(\varepsilon)=t^{*}+\gamma\left(t(\varepsilon)-t^{*}\right) \geq 0, \mathbf{e}^{\top}\left(t^{*}+\gamma l(\varepsilon)\right)=1 \quad \forall \gamma \in\left[0, \gamma_{0}\right] .
$$

For a fixed (sufficiently small) $\varepsilon>0$ define the function

$$
\begin{gathered}
w(\gamma):=\left(t^{*}+\gamma l(\varepsilon)\right)^{\top} \mathcal{A}(z(\varepsilon))\left(t^{*}+\gamma l(\varepsilon)\right)=\left(t^{*}\right)^{\top} \mathcal{A}(z(\varepsilon)) t^{*}+ \\
2 \gamma l(\varepsilon)^{\top} \mathcal{A}(z(\varepsilon)) t^{*}+\gamma^{2} l(\varepsilon)^{\top} \mathcal{A}(z(\varepsilon)) l(\varepsilon)=a \gamma^{2}+2 b \gamma+c \quad \forall \gamma \in\left[0, \gamma_{0}\right],
\end{gathered}
$$

where $c:=\left(t^{*}\right)^{\top} \mathcal{A}(z(\varepsilon)) t^{*} \geq 0, b:=(l(\varepsilon))^{\top} \mathcal{A}(z(\varepsilon)) t^{*}$, and $a:=(l(\varepsilon))^{\top} \mathcal{A}(z(\varepsilon)) l(\varepsilon)$.

By construction, for $\gamma^{*}:=1$ we have $w\left(\gamma^{*}\right)=(t(\varepsilon))^{\top} \mathcal{A}(z(\varepsilon)) t(\varepsilon)$, where $w\left(\gamma^{*}\right)$ is the optimal value of the cost function in the optimization problem

$$
\min t^{\top} \mathcal{A}(z(\varepsilon)) t \quad \text { s.t. } t \in T \text {. }
$$

Thus

$$
w\left(\gamma^{*}\right)=\min _{\gamma \in\left[0, \gamma_{0}\right]} w(\gamma)=\min _{\gamma \in\left[0, \gamma_{0}\right]}\left(a \gamma^{2}+2 b \gamma+c\right)<0 .
$$

As $1=\gamma^{*} \in\left(0, \gamma_{0}\right)$, one can conclude from $(21)$ that $w^{\prime}\left(\gamma^{*}\right)=2\left(a \gamma^{*}+b\right)=0$. Hence, $-a=b$ and we obtain the following equivalent equalities:

$$
\begin{aligned}
& -l(\varepsilon)^{\top} \mathcal{A}(z(\varepsilon)) t^{*}=l(\varepsilon)^{\top} \mathcal{A}(z(\varepsilon)) l(\varepsilon) \Longleftrightarrow \\
& (t(\varepsilon))^{\top} \mathcal{A}(z(\varepsilon)) t^{*}=(t(\varepsilon))^{\top} \mathcal{A}(z(\varepsilon)) t(\varepsilon) .
\end{aligned}
$$

Since $t^{*} \in \operatorname{conv} T_{i m}$, then $t^{*}=\sum_{j \in J} \beta_{j} \tau(j), \quad \sum_{j \in J} \beta_{j}=1, \quad \beta_{j} \geq 0 \forall j \in J$. Hence, taking into account the inclusion $z(\varepsilon) \in \mathcal{X}(\varepsilon) \subset \mathcal{X}$ and the inequality $t(\varepsilon) \geq 0$, we have

$$
(t(\varepsilon))^{\top} \mathcal{A}(z(\varepsilon)) t^{*}=\sum_{j \in J} \beta_{j}(t(\varepsilon))^{\top} \mathcal{A}(z(\varepsilon)) \tau(j) \geq 0 .
$$

The last inequality and inequality (20) contradict equality (22). The lemma is proved.

Lemma 3 For any $\varepsilon>0$ there exists a vector $x(\varepsilon) \in \mathcal{X}$ such that

$$
t^{\top} \mathcal{A}(x(\varepsilon)) t>0 \forall t \in T(\varepsilon) .
$$

Proof For a fixed $\varepsilon>0$, consider the following SIP problem:

$$
\text { (P } \mathrm{PIP}): \quad \max _{x \in \mathbb{R}^{n}, y \in \mathbb{R}} y \quad \text { s.t. } x \in \mathcal{X}, t^{\top} \mathcal{A}(x) t \geq y \quad \forall t \in T(\varepsilon) .
$$

In this problem, the index set $T(\varepsilon)$ is a compact, the set $\mathcal{X}$ defined in (15) is convex, and the constraints satisfy the Slater condition. Then, according to Theorem 1 from [31], there exist an index set $I,|I| \leq n+2$, and vectors

$$
t^{m} \in T(\varepsilon) \forall m \in I,
$$

such that for a discretized problem

$$
\left(\mathrm{P}_{\mathrm{D}}\right): \max _{x \in \mathbb{R}^{n}, y \in \mathbb{R}} y \quad \text { s.t. } x \in \mathcal{X}, \quad\left(t^{m}\right)^{\top} \mathcal{A}(x) t^{m} \geq y \quad \forall m \in I,
$$


it holds $\operatorname{val}\left(\mathrm{P}_{\mathrm{D}}\right)=\operatorname{val}\left(\mathrm{P}_{\mathrm{SIP}}\right)$, where $\operatorname{val}(\mathrm{P})$ denotes the optimal value of the cost function in a problem $(\mathrm{P})$.

Based on the definitions of immobile indices and of the set $T(\varepsilon)$ and taking into account the convexity of the set $\mathcal{X}$, one can show that for the vectors defined in (24), there exists a vector $\widehat{x} \in \mathcal{X}$ such that

$$
\left(t^{m}\right)^{\top} \mathcal{A}(\widehat{x}) t^{m}>0 \forall m \in I .
$$

The last inequalities imply that $\operatorname{val}\left(\mathrm{P}_{\mathrm{D}}\right)>0$ and consequently $\operatorname{val}\left(\mathrm{P}_{\mathrm{SIP}}\right)=\operatorname{val}\left(\mathrm{P}_{\mathrm{D}}\right)>0$. Hence, in the problem $\left(\mathrm{P}_{\mathrm{SIP}}\right)$, there exists a feasible solution $(\bar{x}, \bar{y})$ such that $\bar{y}>0$. The lemma is proved.

\section{Optimality Conditions for Linear Copositive Problems}

In this section, we prove new optimality conditions for the linear copositive problem in form (2). These optimality conditions do not suppose that the constraints of the problem satisfy either the Slater condition or some another additional assumptions and have the form of a criterion.

Theorem 2 Consider problem (2) with $X \neq \emptyset$. Let $T_{i m}$ be the normalized immobile index set and $\{\tau(j), j \in J\}$ be the set of the vertices of the convex polyhedron conv $T_{i m}$. A vector $x^{0} \in X$ is an optimal solution of problem (2) iff there exist vectors

$$
\lambda(j) \in \mathbb{R}_{+}^{p} \forall j \in J, \quad t(i) \in \mathbb{R}_{+}^{p} \forall i \in I \quad \text { with }|I| \leq n,
$$

such that for $x^{0}$ and the matrix

$$
\Omega=\sum_{i \in I} t(i)(t(i))^{\top}+\sum_{j \in J} \tau(j)(\lambda(j))^{\top},
$$

the following relations hold:

$$
-c_{m}+\Omega \bullet A_{m}=0 \forall m=1,2, \ldots, n ; \Omega \bullet \mathcal{A}\left(x^{0}\right)=0 .
$$

Proof Necessity. Let $x^{0} \in X$ be an optimal solution of problem (2).

Consider a perturbed SIP problem

$$
\min _{z \in \mathbb{R}^{n}} c^{\top} z, \quad \text { s.t. } z \in \mathcal{X}\left(\varepsilon_{0}\right),
$$

where the set $\mathcal{X}\left(\varepsilon_{0}\right)$ is defined in (19) and $\varepsilon_{0}>0$ is as in Lemma 2. This problem can be rewritten as follows:

$$
\min _{z \in \mathbb{R}^{n}} c^{\top} z \quad \text { s.t. } \mathcal{A}(z) \tau(j) \geq 0 \forall j \in J ; t^{\top} \mathcal{A}(z) t \geq 0 \forall t \in T\left(\varepsilon_{0}\right) .
$$

According to Lemma 2, the set of feasible solutions of the problem (28) coincides with the set of feasible solutions in the original linear copositive problem (2). Therefore, vector $x^{0}$ is optimal in the problem (28) as well.

Notice the following important properties of the problem (28):

- the index set $T\left(\varepsilon_{0}\right)$ is compact,

- the constraints satisfy the Slater condition (see Lemma 3). 
Hence, by reformulating the classical optimality conditions (see, for example, Theorem 5.107 in [7]) for the optimal solution $x^{0}$ of problem (28), we can state that there exist numbers and vectors

$$
y(i)>0, \quad \eta(i) \in T\left(\varepsilon_{0}\right) \forall i \in I,|I| \leq n ; \quad \lambda(j) \in \mathbb{R}_{+}^{p} \forall j \in J,
$$

such that the following relations hold:

$$
\begin{gathered}
-c_{m}+\sum_{i \in I} y(i)(\eta(i))^{\top} A_{m} \eta(i)+\sum_{j \in J}(\lambda(j))^{\top} A_{m} \tau(j)=0 \forall m=1, \ldots, n ; \\
(\eta(i))^{\top} \mathcal{A}\left(x^{0}\right) \eta(i)=0 \forall i \in I ; \quad(\lambda(j))^{\top} \mathcal{A}\left(x^{0}\right) \tau(j)=0 \forall j \in J .
\end{gathered}
$$

Denoting $t(i):=\sqrt{y(i)} \eta(i)$ for all $i \in I$, it is easy to see that relations (29) can be written in form (27) with the matrix $\Omega$ defined in (26). The necessity is proved.

Sufficiency. Suppose that for $x^{0} \in X$, there exist vectors (25) such that relations (27) hold true for matrix (26). Then, it is easy to show that vector $x^{0}$ is an optimal solution in the following linear programming problem:

$$
\text { (LP) : } \quad \min _{x} c^{\top} x \quad \text { s.t. } \mathcal{A}(x) \tau(j) \geq 0 \forall j \in J ; \quad(t(i))^{\top} \mathcal{A}(x) t(i) \geq 0 \forall i \in I .
$$

It is evident that the set of feasible solutions $X$ of the original problem (2) is a subset of the set of feasible solutions of the problem (LP). Hence, the optimality of $x^{0} \in X$ in the problem (LP) implies the optimality of $x^{0}$ in the original problem (2). The theorem is proved.

Given any symmetric matrix $A \in \mathcal{S}^{p}$ and vectors $l \in \mathbb{R}^{p}, r \in \mathbb{R}^{p}$, evidently we have $r l^{\top} \bullet A=\frac{1}{2}\left(r l^{\top}+l r^{\top}\right) \bullet A$. Then, without loss of generality, we can consider that in (27) the matrix $\Omega$ is symmetric and has the form

$$
\Omega=\sum_{i \in I} t(i)(t(i))^{\top}+\sum_{j \in J}\left(\tau(j)(\lambda(j))^{\top}+\lambda(j)(\tau(j))^{\top}\right) .
$$

Denote by $\mathbb{R}_{+}^{p \times q}$ the set of $p \times q$ matrices with non-negative coefficients and with $q:=$ $|J|$ and consider the matrix $Q=(\tau(j), j \in J) \in \mathbb{R}^{p \times q}$, where as before, $\tau(j), j \in J$, are the vertices of conv $T_{i m}$. Having introduced the set

$$
\Xi(p)=\left\{V \in \mathcal{S}^{p}: V=B Q^{\top}+Q B^{\top}, B \in \mathbb{R}_{+}^{p \times q}\right\},
$$

we can reformulate Theorem 2 as follows.

Theorem 3 A vector $x^{0} \in X$ is an optimal solution of the linear copositive problem (2) iff there exist matrices $U^{0} \in \mathcal{C P}$ and $V^{0} \in \Xi(p)$ such that

$$
-c_{m}+\left(U^{0}+V^{0}\right) \bullet A_{m}=0 \quad \forall m=1,2, \ldots, n ; \quad\left(U^{0}+V^{0}\right) \bullet \mathcal{A}\left(x^{0}\right)=0 .
$$

Here, as before, $\mathcal{C P}=\operatorname{conv}\left\{t t^{\top}: t \in \mathbb{R}_{+}^{p}\right\}$.

The main result of this section is Theorem 2 (and the equivalent Theorem 3). This theorem is outwardly similar to Theorem 3.2 from our recent paper [23]. But the difference between the statements of these theorems and the areas of their application is significant.

Let us consider the following assumption that was done in [23].

Finiteness assumption: The normalized immobile index set is either empty or consists of a finite number of elements: $\left|T_{i m}\right|<\infty$.

This assumption lays in the basis of the results obtained in [23] since

- the optimality criterion (Theorem 3.2) is proved in [23] using the results of [21], where the Finiteness assumption is essential; 
- $\quad$ Theorem 3.2 and the duality results are formulated and proved in [23] using explicitly all elements from the index set $T_{i m}$.

Therefore, it is evident that the optimality conditions for linear copositive programming formulated in [23] cannot be used in the case when the set $T_{i m}$ contains the continuum of elements.

Notice that in the general case, the set $T_{i m}$ can contain an infinite number of elements (see Lemma 1 and the example below).

In this paper, for the linear copositive problem (2), we have proved new optimality criteria (Theorems 2 and 3) without making the Finiteness assumption (as well as any other assumption) and not using the outcomes of [21]. This permits us to conclude that to apply these optimality results as well as the subsequent duality formulations (see Section 5), one does not need to know all the elements of the (possibly infinite) set $T_{i m}$. It is sufficient just to find a finite number of some elements of this set, namely the vertices $\tau(j), j \in J$, of the polyhedron conv $T_{i m}$.

\section{Example}

In [23], for linear copositive problems satisfying the Finiteness assumption, we compared the obtained optimality conditions with other optimality conditions known from the literature and showed that there are situations where only the optimality conditions from [23] permit to reveal the optimality of solutions of these problems. Evidently, this conclusion can be transferred to the optimality conditions obtained in this paper.

In this section, we will test optimality conditions on an example where the set $T_{i m}$ consists of the continuum of elements.

Let us consider problem (2) with the following data: $n=7, \quad p=5$,

$$
\begin{aligned}
& A_{0}=\frac{1}{2}\left(\begin{array}{rrrrr}
2 & -2 & 1 & 2 & -2 \\
-2 & 2 & -1 & 1 & 2 \\
1 & -1 & 2 & -2 & 2 \\
2 & 1 & -2 & 2 & -2 \\
-2 & 2 & 2 & -2 & 2
\end{array}\right), A_{1}=\left(\begin{array}{rrrrr}
0 & -1 & 0 & 0 & 0 \\
-1 & -2 & 0 & 0 & 0 \\
0 & 0 & 4 & -1 & 0 \\
0 & 0 & -1 & 0 & 0 \\
0 & 0 & 0 & 0 & 0
\end{array}\right), A_{2}=\left(\begin{array}{rrrrr}
0 & 0 & 1 & 0 & 0 \\
0 & 4 & 0 & 3 & 0 \\
1 & 0 & -2 & 0 & 0 \\
0 & 3 & 0 & 0 & 0 \\
0 & 0 & 0 & 0 & 0
\end{array}\right), \\
& A_{3}=\left(\begin{array}{rrrrr}
0 & 1 & -1 & 0 & 0 \\
1 & -2 & 1 & 0 & 0 \\
-1 & 1 & -2 & 1 & 0 \\
0 & 0 & 1 & 0 & 0 \\
0 & 0 & 0 & 0 & 0
\end{array}\right), A_{4}=\left(\begin{array}{rrrrr}
0 & -1 & 0 & 0 & 0 \\
-1 & 0 & 0 & 0 & 0 \\
0 & 0 & 2 & 0 & 0 \\
0 & 0 & 0 & 0 & 0 \\
0 & 0 & 0 & 0 & 0
\end{array}\right), A_{7}=\frac{1}{2}\left(\begin{array}{rrrrr}
0 & 0 & 1 & 0 & 0 \\
0 & 0 & -1 & 1 & 0 \\
1 & -1 & 0 & 0 & 0 \\
0 & 1 & 0 & 0 & 0 \\
0 & 0 & 0 & 0 & 0
\end{array}\right), \\
& A_{5}=e_{1} e_{2}^{\top}+e_{2} e_{1}^{\top}, A_{6}=A_{1}-A_{2}+A_{3}-A_{4}+A_{5}, c=(-6,4,2,-3,3,-2,0)^{\top},
\end{aligned}
$$

where $e_{i} \in \mathbb{R}^{5}$ denotes the $i$-th basic vector of the canonic basis of $\mathbb{R}^{5}$.

For a given symmetric matrix $D$, denote

$$
\mathcal{V}^{D}:=\left\{t \in \mathbb{R}_{+}^{p}: t^{\top} D t=0, \quad \mathbf{e}^{\top} t=1\right\} .
$$

Consider vectors

$$
\begin{gathered}
v(1)=\frac{1}{2}(1,0,0,0,1)^{\top} ; v(2)=\frac{1}{2}(1,1,0,0,0)^{\top}, v(3)=\frac{1}{2}(0,1,1,0,0)^{\top}, \\
v(4)=\frac{1}{2}(0,0,1,1,0)^{\top}, v(5)=\frac{1}{2}(0,0,0,1,1)^{\top},
\end{gathered}
$$


and the set $\Delta:=\operatorname{conv}\{v(1), v(5)\}$.

One can show that $\mathcal{V}^{A_{0}}=\left(\bigcup_{i=1}^{4} \operatorname{conv}\{v(i), v(i+1)\}\right) \cup \Delta$ and

$$
v(1)^{\top} A_{m} v(1)=v(1)^{\top} A_{m} v(5)=v(5)^{\top} A_{m} v(5)=0 \quad \forall m=0,1, \ldots, 7 \text {. }
$$

Hence for any $x \in \mathbb{R}^{7}$, we have $t^{\top} \mathcal{A}(x) t=0 \quad \forall t \in \Delta$ and may conclude that $\Delta \subset T_{i m}$. Then, evidently, the set $T_{i m}$ is infinite.

For this example, set $\mathcal{B}(x):=\sum_{m=1}^{7} A_{m} x_{m}$. Then $\mathcal{A}(x)=\mathcal{B}(x)+A_{0}$.

Consider vector $\bar{x}=(2,3,2,2,2,0,0)^{\top}$ and calculate $\mathcal{B}(\bar{x})=\left(\begin{array}{lllll}0 & 0 & 1 & 0 & 0 \\ 0 & 4 & 2 & 9 & 0 \\ 1 & 2 & 2 & 0 & 0 \\ 0 & 9 & 0 & 0 & 0 \\ 0 & 0 & 0 & 0 & 0\end{array}\right)$.

It is evident that $\mathcal{B}(\bar{x}) \in \mathcal{C O} \mathcal{P}^{5}$. Taking into account that $A_{0} \in \mathcal{C O} \mathcal{P}^{5}$ (see [12]), we get that $\mathcal{A}(\bar{x}) \in \mathcal{C O} \mathcal{P}^{5}$, and hence $\bar{x}$ is a feasible solution of our problem. One can check that $\mathcal{V}^{\mathcal{B}(\bar{x})}=\operatorname{conv}\left\{e_{1}, e_{4}, e_{5}\right\}$. Consequently, $\mathcal{V}^{\mathcal{A}(\bar{x})}=\mathcal{V}^{\mathcal{B}(\bar{x})} \cap \mathcal{V}^{A_{0}}=\Delta$. This implies that $T_{i m}=\Delta$.

The vertices of the polyhedron conv $T_{i m}=T_{i m}$ are

$$
\tau(1):=v(1)=\frac{1}{2}(1,0,0,0,1)^{\top}, \tau(2):=v(5)=\frac{1}{2}(0,0,0,1,1)^{\top} .
$$

Hence $J=\{1,2\}$ (see formula (13)).

According to Proposition 2, for all feasible $x$ in this problem, the following inequalities are satisfied: $\mathcal{A}(x) \tau(1) \geq 0, \mathcal{A}(x) \tau(2) \geq 0$.

Now, consider vector $x^{0}=(1,1,1,1,1,0,0)^{\top}$ and the corresponding matrix $\mathcal{A}\left(x^{0}\right)=$ $\mathcal{B}\left(x^{0}\right)+A_{0}$, where $\mathcal{B}\left(x^{0}\right)=e_{2} e_{3}^{\top}+e_{3} e_{2}^{\top}+3\left(e_{2} e_{4}^{\top}+e_{4} e_{2}^{\top}\right)+2 e_{3} e_{3}^{\top}$.

Since $\mathcal{B}\left(x^{0}\right) \in \mathcal{C O} \mathcal{P}^{5}$ and $A_{0} \in \mathcal{C O} \mathcal{P}^{5}$ we conclude that $\mathcal{A}\left(x^{0}\right) \in \mathcal{C O} \mathcal{P}^{5}$ and $x^{0}$ is a feasible solution in our problem.

One can check that $\mathcal{V}^{\mathcal{B}\left(x^{0}\right)}=\operatorname{conv}\left\{e_{1}, e_{2}, e_{5}\right\} \cup \operatorname{conv}\left\{e_{1}, e_{4}, e_{5}\right\}$. Consequently, $\mathcal{V}^{\mathcal{A}\left(x^{0}\right)}=\mathcal{V}^{\mathcal{B}(x)} \cap \mathcal{V}^{A_{0}}=\Delta \cup \operatorname{conv}\{v(1), v(2)\}$.

It can be easily verified that vector $x^{0}$ is an optimal solution of the following linear programming problem:

$$
\min c^{\top} x \text {, s.t. } \mathcal{A}(x) \tau(1) \geq 0, \mathcal{A}(x) \tau(2) \geq 0,(v(2))^{\top} \mathcal{A}(x) v(2) \geq 0 .
$$

Taking into account that the set of feasible solutions of the linear copositive problem in our example belongs to the set of feasible solutions of this linear programming problem, we conclude that $x^{0}$ is an optimal in the copositive problem as well.

For the given $x^{0}$, the optimality conditions (27) of Theorem 2 are satisfied with matrix $\Omega$ in the form (26) calculated for the sets and vectors

$$
I=\{1\}, J=\{1,2\}, \quad t(1)=2 v(2), \lambda(1)=2 e_{2}, \lambda(2)=2 e_{3} .
$$

To justify that the optimality criteria (CQ-free optimality conditions) proved in Section 3 of this paper (Theorem 2), are more general than the optimality conditions proved in [1] (Theorem 1), we show that the optimality conditions formulated in Theorem 1, do not allow one to recognize the optimality of the vector $x^{0}$. 
For the given $x^{0}$, the conditions (KKT) of Theorem 1, consist in existence of such a matrix $\Omega \in \mathcal{C} \mathcal{P}^{5}=\operatorname{conv}\left\{t t^{\top}: t \in \mathbb{R}_{+}^{5}\right\}$ that

$$
\text { (a) : }-c_{m}+\Omega \bullet A_{m}=0 \forall m=1, \ldots, 7 ; \quad \text { (b) }: \Omega \bullet \mathcal{A}\left(x^{0}\right)=0 .
$$

From condition (b), it follows that for some finite set $\Upsilon \subset \mathbb{N},|\Upsilon|<\infty$, matrix $\Omega$ has the form $\Omega=\sum_{i \in \Upsilon} \eta(i)(\eta(i))^{\top}$, where $\eta(i) \in R_{a}\left(x^{0}\right) \forall i \in \Upsilon$. Here and in what follows, given $x \in X$, we will refer to the set $R_{a}(x)$ defined as

$$
R_{a}(x):=\left\{t \in \mathbb{R}_{+}^{p}: t^{\top} \mathcal{A}(x) t=0\right\}=\left\{t=\alpha \tau, \tau \in \mathcal{V}^{\mathcal{A}(x)}, \alpha \geq 0\right\},
$$

as to the set of active in $x$ indices (the active index set) in problem (2).

It follows from (33) that $t^{\top} A_{m} t=0$ for all $m=0,1, \ldots, 7$ and all $t \in \Delta$. Hence

$\Omega=\sum_{i \in \Upsilon} \alpha_{i} \eta(i)(\eta(i))^{\top}$, where $\alpha_{i}>0, \eta(i) \in \operatorname{conv}\{v(1), v(2)\}$ for all $i \in \Upsilon$. Then the conditions (a) from (35) take the form

$$
c_{m}=y_{1} v(1)^{\top} A_{m} v(1)+y_{2} v(1)^{\top} A_{m} v(2)+y_{2} v(2)^{\top} A_{m} v(2) \forall m=1, \ldots, 7
$$

with some coefficients $y_{1} \geq 0, y_{2} \geq 0, y_{3} \geq 0$. It is easy to check that in our example this system is inconsistent.

Thus, we have shown that the (sufficient) optimality conditions formulated in Theorem 1 are not able to recognize the optimality of the vector $x^{0}$ in this problem (the conditions (KKT) are not satisfied).

Consider vectors $x^{*}=(-1,1,-1,1,-1,1,0)^{\top}, \tilde{x}=(0,0,0,0,0,0,1)^{\top}$, and the corresponding matrices

$$
\mathcal{A}\left(x^{*}\right)=A_{0}, \quad \mathcal{A}(\tilde{x})=\left(\begin{array}{rrrrr}
1 & -1 & 1 & 1 & -1 \\
-1 & 1 & -1 & 1 & 1 \\
1 & -1 & 1 & -1 & 1 \\
1 & 1 & -1 & 1 & -1 \\
-1 & 1 & 1 & -1 & 1
\end{array}\right) .
$$

It is known (see [12]) that $\mathcal{A}\left(x^{*}\right) \in \mathcal{C} \mathcal{O} \mathcal{P}^{5}, \mathcal{A}(\tilde{x}) \in \mathcal{C O} \mathcal{P}^{5}$. Since $c^{\top} x^{*}=c^{\top} \tilde{x}=c^{\top} x^{0}$, one can conclude that the vectors $x^{*}$ and $\tilde{x}$ are optimal solutions in our linear copositive problem.

For $x^{*}$ and $\tilde{x}$, the optimality conditions (27) of Theorem 2 are satisfied with matrix in the form (26) and data (34).

Now, we will test whether or not the conditions of Theorem 1 are satisfied for these optimal solutions.

Consider, first, $x^{*}$. Since the active index set in $x^{*}$ has the form

$$
R_{a}\left(x^{*}\right)=\left\{\alpha t: t \in \mathcal{V}^{A_{0}}, \alpha \geq 0\right\},
$$

it follows from the condition (b) in (35) that for some finite set $\Upsilon$, it holds $\Omega=$ $\sum_{i \in \Upsilon} \eta(i)(\eta(i))^{\top}$, where $\eta(i) \in R_{a}\left(x^{*}\right)$ for all $i \in \Upsilon,|\Upsilon|<\infty$.

Hence, the conditions (a) in (35) take the form

$$
c_{m}=\sum_{i \in \Upsilon} \alpha_{i} \eta(i)^{\top} A_{m} \eta(i) \quad \forall m=1, \ldots, 7,
$$

where $\alpha_{i}>0, \eta(i) \in \mathcal{V}^{A_{0}} \forall i \in \Upsilon$.

Recall that in our problem there exists another optimal solution $x^{0}$. Then it is easy to show that equalities (38) and the inequality $\alpha_{i}>0$ imply the inclusion $\eta(i) \in \mathcal{V}^{A_{0}} \cap$ 
$\mathcal{V}^{\mathcal{A}\left(x^{0}\right)}=\mathcal{V}^{\mathcal{A}\left(x^{0}\right)}$. But above we have shown that the system (37) is inconsistent. Hence the conditions of Theorem 1 are not satisfied for the optimal solution $x^{*}$ as well.

In a similar way one can show that the conditions of Theorem 1 are not satisfied for the optimal solution $\tilde{x}$ (as well as for any other optimal solution).

Notice that in this example, the set

$$
X_{\text {opt }}^{*}=\left\{x=\alpha_{1} x^{0}+\alpha_{2} x^{*}+\alpha_{3} \tilde{x} \forall \alpha_{1} \geq 0, \forall \alpha_{2} \geq 0, \forall \alpha_{3} \in[0,1]\right\}
$$

belongs to the set of optimal solutions $X_{\text {opt }}$ in our linear copositive problem.

Summing up, we can state that in this example

- the normalized immobile index set $T_{i m}$ consists of an infinite number of elements;

- for all optimal solutions $x \in X_{o p t}^{*}$, the corresponding index sets $\mathcal{V}^{\mathcal{A}(x)}$ are bigger than the set $T_{i m}$;

- all optimal solutions of the linear copositive problem satisfy the optimality conditions (27) formulated in Theorem 2 with matrix in the form (26) and data (34) and do not satisfy the sufficient optimality conditions of Theorem 1;

- the linear copositive problem considered in the example cannot be reduced to an SDP problem since for all optimal solutions in the form $y=\alpha_{1} x^{*}+\alpha_{2} \tilde{x}, \alpha_{1} \geq 0, \alpha_{2} \in[0,1]$, it holds $\mathcal{A}(y) \notin \mathcal{S}_{+}^{5}+\mathcal{N}^{5}$ (see Theorem 3.1 in [12]). Here $\mathcal{S}_{+}^{p}, p \in \mathbb{N}$, denotes the cone of $p \times p$ symmetric positive semidefined matrices and $\mathcal{N}^{p}$ stays for the set of all $p \times p$ symmetric entrywise nonnegative matrices;

- the dimension of the set aff $\left\{x \in X_{o p t}: \mathcal{A}(x) \notin \mathcal{S}_{+}^{5}+\mathcal{N}^{5}\right\}$ is equal to or more than 2 . Here aff $\mathcal{D}$ denotes the affine hull of a set $\mathcal{D}$.

All the above confirms our conclusion about the importance and novelty of the optimality conditions obtained in Section 3. Being criteria, these conditions permit to detect the optimality of optimal solutions of copositive problems even in the situations when other known (sufficient) optimality conditions fail. Such strong results were possible thanks to the efficient use of the set of immobile indices.

In the rest of the paper, we will show how the approach presented in the paper can be used to obtain new strong dual formulations for copositive programming.

\section{Dual Formulations of Copositive Problems: the Standard Lagrangian Dual and the Regularized Dual Problems}

In this section, we will discuss some new dual formulations for the problem (2). Duality aspects are of high importance in study of optimization problem. Duality plays a central role in detecting infeasibility, lower-bounding the optimal objective value, as well as in design and analysis of iterative algorithms. In [37, 38], a CQ-free duality theory for conic optimization was developed in terms of so-called minimal cone. Being quite general, this theory has one disadvantage in terms of its application, namely, it is very abstract. Having in our disposal the optimality criterion in the form of Theorem 3, now, in a similar way as it was done in [23], it is easy to deduce for linear copositive problems new strong dual formulations without constraint qualifications and/or other additional assumptions. To obtain these results, we will use Theorem 3 and the same reasoning scheme as in [23]. For the sake of completeness, let us bring this reasoning here.

The (standard) Lagrangian dual problem for (2) is as follows [1]:

$$
\max _{W}\left(-W \bullet A_{0}\right) \quad \text { s.t. }-c_{i}+W \bullet A_{i}=0, i=1,2, \ldots, n ; W \in \mathcal{C P},
$$


where, as above, the cone $\mathcal{C P}=\operatorname{conv}\left\{l l^{\top}: l \in \mathbb{R}_{+}^{p}\right\}$ is dual to $\mathcal{C O P}$.

It is well known (see Theorem 3.1 in [1]) that if the constraints of problem (2) satisfy the Slater condition, then there is no gap between the optimal values of problems (2) and (39).

If the constraints of problem (2) do not satisfy the Slater condition, then the positive gap is possible. Notice that it may happen even in the case when problem (2) has an optimal solution.

Let $T_{i m}$ be the normalized immobile index set in problem (2) and $\{\tau(j), j \in J\}$ be the set of vertices of the polyhedron conv $T_{i m}$. Recall that $|J|<\infty$.

Consider the following closed cones:

$$
K(j):=\left\{D \in \mathcal{S}^{p}: D \tau(j) \geq 0\right\}, \quad j \in J .
$$

Observe that all cones $K(j), j \in J$, as well as the cone of copositive matrices $\mathcal{C O P}$ defined in (1), are convex and closed. The cones $K(j), j \in J$, are explicitly generated by the finite index set $\{\tau(j), j \in J\}$.

It is easy to show that for any $j \in J$, the dual cone to $K(j)$ has the form

$$
K^{*}(j)=\left\{l(\tau(j))^{\top}+\tau(j) l^{\top}: l \in \mathbb{R}_{+}^{p}\right\} .
$$

Here and in what follows, for a given cone $\mathcal{C} \subset \mathbb{R}^{p}$, we denote by $\mathcal{C}^{*}$ its dual (polar) cone defined by $\mathcal{C}^{*}:=\left\{u \in \mathbb{R}^{p}: u^{\top} v \geq 0 \forall v \in \mathcal{C}\right\}$.

Denote

$$
\mathcal{K}:=\left(\bigcap_{j \in J} K(j)\right) \cap \mathcal{C O} \mathcal{P} .
$$

It is known [4, 5] that given a family of closed convex cones $\mathcal{E}_{i}, i=1, \ldots, m$, it holds $\left(\bigcap_{i=1}^{m} \mathcal{E}_{i}\right)^{*}=\mathrm{cl}\left(\sum_{i=1}^{m} \mathcal{E}_{i}^{*}\right)$. Hence, the dual cone to $\mathcal{K}$ has the form

$$
\mathcal{K}^{*}=\operatorname{cl}\left(\sum_{j \in J} K^{*}(j)+\mathcal{C P}\right) .
$$

Taking into account Proposition 2, one can show that the original linear copositive problem in form (2) is equivalent to the following problem in a conic form:

$$
\min _{x \in \mathbb{R}^{n}} c^{\top} x \quad \text { s.t. } \mathcal{A}(x) \in \mathcal{K} \subset \mathcal{C O} \mathcal{P} .
$$

Let us refer to problem (40) as to a regularized primal problem.

Its dual (the regularized dual problem) has the form

$$
\max _{W}\left(-W \bullet A_{0}\right) \quad \text { s.t. }-c_{m}+W \bullet A_{m}=0 \quad \forall m=1,2, \ldots, n, W \in \mathcal{K}^{*} .
$$

It can be shown that given any feasible solution $x$ of problem (40) and any feasible solution $W$ of the dual problem (41), the following inequality (weak duality) holds:

$$
c^{\top} x \geq-W \bullet A_{0} .
$$

Let us show that for any optimal solution $x^{0}$ of problem (40), there exists a feasible solution $W^{0}$ of the dual problem (41) such that the strong duality property holds:

$$
c^{\top} x^{0}=-W^{0} \bullet A_{0} .
$$

Indeed, according to Theorem 3, for any optimal solution $x^{0}$ of the linear copositive problem (2) (and, hence, of the regularized primal problem (40)) there exist matrices $U^{0} \in$ $\mathcal{C P}$ and $V^{0} \in \Xi(p)$ such that conditions (32) are fulfilled. The set $\Xi(p)$ is defined in (31). 
Let us set $W^{0}:=U^{0}+V^{0}$. It is easy to see that by construction, $W^{0} \in \mathcal{K}^{*}$ and due to relations (32) we have

$$
\begin{gathered}
-c_{m}+W^{0} \bullet A_{m}=0 \forall m=1,2, \ldots, n, \\
W^{0} \bullet \mathcal{A}\left(x^{0}\right)=0 .
\end{gathered}
$$

It follows from the inclusion $W^{0} \in \mathcal{K}^{*}$ and equalities (43) that the matrix $W^{0}$ is a feasible solution of the dual problem (41). Moreover, it is easy to see that equalities (43), (44) imply equality (42).

Thus, we have proved the following proposition.

Proposition 4 Suppose that the primal linear copositive problem (2) has an optimal solution. Then

- $\quad$ an optimal solution of the regularized dual problem (41) exists, and

- there is no gap between the optimal values of problem (2) (or, equivalently (40)), and its regularized dual problem (41).

From the proposition above, it follows that the strong duality is guaranteed for the primal problem (2) and its regularized dual problem (41), while, as it was mention above, for the pair constituted by the primal problem (2) and its (standard) Lagrangian dual problem (39), the strong duality may fail.

In [23], we presented an example (see section 4 of [23]) that illustrated these conclusions for the case $\left|T_{i m}\right|=1$. Taking into account that the Slater condition is equivalent to the condition $\left|T_{i m}\right|=0$, one can conclude that in the example, a minimal violation of the Slater CQ occurs.

The main contribution of this section is to obtain for the copositive problem (2) a new regularized dual one (41) satisfying the strong duality conditions without CQs or any other assumptions. The dual problem (41) has explicit form and is constructed using the vertices of the convex hull of the normalized immobile index set of the constraints in the primal linear copositive problem (2). Proposition 4 guarantees zero duality gap.

For comparison of the new conic formulations obtained using the notion of the immobile indices with that from [1, 9, 38], we refer the interested reader to the paper [23].

We would like to mention that in [36], a so-called extended Lagrange-Slater dual program (ELSD), was formulated for SDP problems, and it was shown that the obtained pair of dual SDP problems satisfies the strong duality. The ELSD problem was constructed with the help of a special technique which permitted to express the feasible set of the Lagrange multipliers as a solution of a finite system of constraints. In future, for linear copositive problems, we intend to develop a similar technique which may permit to obtain strong dual formulations without explicit usage of the normalized immobile index set $T_{i m}$ as well as the vertices of its convex hull.

\section{Conclusions and the Future Work}

The main aim of this paper was to strengthen the optimality results obtained for linear copositive problems in [23], namely, to prove the conjecture that the new optimality conditions based on the notion of the immobile indices, hold true without additional assumptions such as, for example, the finiteness assumption. This goal has been fully achieved by introducing the normalized immobile index set and in-depth exploration of its properties. 
In the paper, we formulated and proved new CQ-free optimality conditions that use the finite number of the vertices of the convex hull of the normalized immobile index set instead of the immobile indices themselves as it was done in [23]. This permitted to test the optimality in the situations when the conditions from [23], as well as that from [1], cannot be used. The developed in the paper approach permitted us to obtain for the copositive problem (2) a new regularized dual one (41) satisfying the strong duality conditions without CQs or any other assumptions. The regularized dual problem has explicit form and closes the duality gap for linear copositive problems.

The results of the paper confirm that the new approach to optimality conditions based on the notion of immobile indices is a powerful tool in studying the optimality and duality not only in semi-infinite and semidefinite programming, but can also be applied to a new and less studied area such as copositive optimization.

Based on the proposed approach, there are several directions of future research, among them the following:

- building constructive procedures for finding the vertices of the convex hull of the normalized immobile index set;

- applying the results of the paper to bring out new optimality conditions for different classes of convex optimization problems;

- creating numerical methods based on the immobile indices for different classes of convex optimization problems which admit copositive and conic reformulations;

- developing new approaches to solving copositive problems based on the strong duality results obtained in the paper;

- establishing deep no-gap duality theory based on the notion of immobile indices.

Given the importance of applications of robust optimization, it would also be interesting to apply the new approach to optimization problems with different forms of uncertainty, such as LP problems with conic uncertainty, robust cone programs and others (for the modern aspects of robust optimization and optimization under uncertainty see e.g. $[3,39])$.

Acknowledgements This work has been supported by the state research program "Convergence" (Republic Belarus), Task 1.3.01 (O.I. Kostyukova), by Portuguese funds through CIDMA - Center for Research and Development in Mathematics and Applications, and FCT - Portuguese Foundation for Science and Technology, within the project UID/MAT/04106/2019 (T.V.Tchemisova), and by the RUDN University Program 5-100 (O.S.Dudina).

The authors thank the anonymous referees for their valuable comments on this paper.

\section{References}

1. Ahmed, F., Dür, M., Still, G.: Copositive programming via semi-infinite optimization. J. Optim. Theory Appl. 159, 322-340 (2013)

2. Baumer, L.D.: Extreme copositive quadratic forms. Pac. J. Math. 19(2), 197-204 (1966)

3. Ben-Tal, A., Ghaoui, L.E., Nemirovski, A.: Robust Optimization. Princeton University Press (2009)

4. Berman, A., Shaked-Monderer, N.: Completely Positive Matrices. World Scientific, New Jersey (2003). $216 \mathrm{p}$

5. Bomze, I.M., Dür, M., de Klerk, E., Roos, C., Quist, A.J., Terlaky, T.: On copositive programming and standard quadratic optimization problems. J. Global Optim. 18, 301-320 (2000)

6. Bomze, I.M.: Copositive optimization - recent developments and applications. EJOR 216(3), 509-520 (2012)

7. Bonnans, J.F., Shapiro, A.: Perturbation Analysis of Optimization Problems. Springer, New-York (2000). $601 \mathrm{p}$ 
8. Burer, S.: On the copositive representation of binary and continuous nonconvex quadratic programs. Math. Program 120, 479-495 (2009)

9. Borwein, J.M., Wolkowicz, H.: Characterization of optimality for the abstract convex program with finite-dimensional range. J. Aust. Math. Soc., Ser. A 30(4), 390-411 (1980)

10. Cánovas, M.J., Kruger, A.Y.a., López, M.A., Parra, J., Théra, M.A.: Calmness modulus of linear semiinfinite programs. SIAM J. Optim. 24(1), 29-48 (2014)

11. de Klerk, E., Pasechnik, D.V.: Approximation of the stability number number of a graph via copositive programming. SIAM J. Optim. 12, 875-892 (2002)

12. Dickinson, P.J., Dür, M., Gijben, L., Hildebrand, R.: Irreducible elements of the copositive cone. Linear Algebra Appl. 439(6), 1605-1626 (2013)

13. Dickinson, P.J.C., Hildebrand, R.: Considering copositivity locally. J. Math. Anal. Appl. 437(2), 11841195 (2016)

14. Dür, M.: Copositive Programming - a Survey. In: Diehl, M., Glineur, F., Jarlebring, E., Michielis, W. (eds.) Recent Advances in Optimization and its Applications in Engineering, p. 535 p. Springer, Berlin (2010)

15. Jongen, H.T.h., Twilt, F., Weber, G.-W.: Semi-infinite optimization: Structure and stability of the feasible set. J. Optim. Theory Appl. 72, 529-552 (1992)

16. Hiriart-Urruty, J.B., Seeger, A.: A variational approach to copositive matrices. SIAM Rev. 52, 593-629 (2010)

17. Hettich, R., Kortanek, K.O.: Semi-Infinite programming: Theory, methods and applications. SIAM Rev. 35, 380-429 (1993)

18. Hildebrand, R.: Minimal zeros of copositive matrices. Linear Algebra Appl. 459, 154-174 (2014)

19. Kostyukova, O.I., Tchemisova, T.V.: Convex SIP problems with finitely representable compact index sets: Immobile indices and the properties of the auxiliary NLP problem. Set-Valued Var. Anal. 23(3), 519-546 (2015)

20. Kostyukova, O.I., Tchemisova, T.V.: Optimality conditions for convex semi-infinite programming problems with finitely representable compact index sets. J. Optim. Theory Appl. 175(1), 76-103 (2017)

21. Kostyukova, O.I., Tchemisova, T.V.: On a constructive approach to optimality conditions for convex SIP problems with polyhedral index sets. Optimization 63(1), 67-91 (2014)

22. Kostyukova, O.I., Tchemisova, T.V.: Optimality criteria without constraint qualification for linear semidefinite problems. Special Issue "Algebraic techniques in Graph Theory and Optimization”. J. Math. Sci. 182(2), 126-143 (2012)

23. Kostyukova O.I., Tchemisova, T.V.: Optimality conditions for linear copositive programming problems with isolated immobile indices. Optimization 69(1), 145-164 (2020)

24. Kostyukova, O.I., Tchemisova, T.V.: Implicit optimality criterion for convex SIP problem with box constrained index set. TOP 20(2), 475-502 (2012)

25. Kruger, A.Y.a.: Strict $(\varepsilon, \delta)$-subdifferentials and extremality conditions. Optimization 51(3), 539-554 (2002)

26. Kruger, A.Y.a.: Weak stationarity: Eliminating the gap between necessary and sufficient conditions Optimization 53:2, 147-164 (2004)

27. Kruger, A.Y.a.: About regularity of collections of sets. Set-Valued Anal. 14, 187-206 (2006)

28. Kruger, A.Ya.: Generalized differentials of nonsmooth functions and necessary conditions for an extremum. Sib. Math. J. 26, 370-379 (1985)

29. Kruger, A.Ya., Minchenko, L., Outrata, J.V.: On relaxing the Mangasarian-Fromovitz constraint qualification. Positivity 18(1), 171-189 (2014)

30. Kruger, A.Ya., López, M.A.: Stationarity and regularity of infinite collections of sets. J. Optim Theory Appl 154, 339-369 (2012)

31. Levin, V.L.: Application of E. Helly's theorem to convex programming, problems of the best approximation and related questions. Math. USSR Sb. 8(2), 235-247 (1969)

32. Motzkin, T.S., Straus, E.G.: Maxima for graphs and a new proof of a theorem of Turán. Can. J. Math. 17, 533-540 (1965)

33. Murtu, K.G., Kabadi, S.N.: Some NP-complete problems in quadratic and nonlinear programming. Math Program. 39, 117-129 (1987)

34. Ngai, H.V., Kruger, A.Ya., Théra, M.A.: Stability of error bounds for convex constraint systems in Banach spaces. SIAM J. Optim. 20(6), 3280-3296 (2010)

35. Ngai, H.V., Kruger, A.Ya., Théra, M.A.: Stability of error bounds for semi-infinite convex constraint systems. SIAM J. Optim. 20(4), 2080-2096 (2010)

36. Ramana, M.V.: An exact duality theory for semidefinite programming and its complexity implications. Math. Program. 77, 129-162 (1997) 
37. Ramana, M.V., Tuncel, L., Wolkowicz, H.: Strong duality for semidefinite programming. SIAM J. Optim. 7(3), 641-662 (1997)

38. Tunçel, L., Wolkowicz, H.: Strong duality and minimal representations for cone optimization. Comput. Optim. Appl. 53, 619-648 (2013)

39. Weber, G.-W.: Generalized Semi-Infinite Optimization and Related Topics. In: Hofmann, K.H., Wille, R. (eds.) Research and Exposition in Mathematics, vol. 29. Heldermann Publishing House, Lemgo (2003)

40. Weber, G.-W., Tezel, A.: On generalized semi-infinite optimization of genetic networks. TOP 15(1), 65-77 (2007)

41. Yamamoto, S.: Alternative theorems and constraint qualifications in convex optimization. Mem. Gra. Sci. Eng. Shimane Univ. Series B: Math. 50, 11-30 (2017)

Publisher's Note Springer Nature remains neutral with regard to jurisdictional claims in published maps and institutional affiliations. 